\title{
Commentary Understanding genetics of sepsis: will new technology help?
} Sachin Yende ${ }^{1,2}$ and John A Kellum ${ }^{1,2}$

\begin{abstract}
${ }^{1}$ The Clinical Research, Investigation, and Systems Modeling of Acute Illness (CRISMA) Laboratory, University of Pittsburgh, Pittsburgh, PA 15261, USA ${ }^{2}$ Department of Critical Care Medicine, 604 Scaife Hall, University of Pittsburgh School of Medicine, 3550 Terrace Street, Pittsburgh, PA 15261, USA
\end{abstract}

Corresponding author: John A Kellum, kellumja@upmc.edu

Published: 6 May 2009

Critical Care 2009, 13:141 (doi:10.1186/cc7727)

This article is online at http://ccforum.com/content/13/3/141

(C) 2009 BioMed Central Ltd

See related review by Sutherland and Walley, http://ccforum.com/content/13/2/210

\begin{abstract}
High-throughput techniques, such as genome-wide scans, will allow genotyping of a large number of single-nucleotide polymorphisms throughout the human genome. There is intense interest to apply this technology to understand genetics of complex traits, including severe sepsis. To effectively utilize this technology, large cohorts of septic patients will have to be recruited. Careful attention should be paid to different aspects of study design and analyses as large, multicenter cohorts are assembled for genome-wide association studies.
\end{abstract}

Resistance to infection and its outcomes are influenced by a complex interplay between the host, the microbe, and the environment. Severe sepsis occurs due to abnormalities in the host response to infection. There is great interest to understand whether genetic determinants of the host response to infection are associated with poor outcomes of infection. Identifying these genetic determinants could be important to improve performance of current risk prediction models. Furthermore, the pharmacogenomics of sepsis will allow us to target immune-modulating therapies. Despite these plausible benefits and more than a decade of research, the role of genetic determinants in severe sepsis remains unclear. Results of some studies have been encouraging, but these results have not been replicated consistently in subsequent, larger studies.

In the previous issue of Critical Care, Sutherland and Walley provide a broad overview of gene-association studies, the most common study design to assess genetics of infection and sepsis [1]. The review highlights genome-wide association studies (GWAS), an important and emerging technology to understand genetics of complex traits. Highdensity SNP platforms by Affymetrix (Santa Clara, CA, USA) and Illumina (San Diego, CA, USA) allow genotyping of up to one million SNPs. Imputation methods using linkage patterns between SNPs genotyped using these platforms and data from the International Hapmap project or prior results of sequencing allow us to estimate additional unmeasured genotypes [2]. This method increases the power and allows comparison of results across different platforms. Up to two million SNPs can therefore be analyzed using GWAS, which may allow discovery of proteins that have not been studied in the sepsis pathophysiology.

Similar to any new technology, the advantage of genotyping a large number of polymorphisms poses unique challenges during statistical analysis. For instance, given the sheer number of variables that are tested, the traditional $P$ value cutoff points are clearly inadequate - and even $P<10^{-6}$ may include a large number of false positives [3]. Furthermore, since the relative risk of individual polymorphisms is likely to be small, analyzing large samples will be necessary for GWAS. Several thousand subjects should therefore be included in these studies. A glance at Table 1 in Sutherland and Walley's article shows that current studies assessing genetics of sepsis have been much smaller [1]. Finally, replication is critical to confirm these results - often in several cohorts, requiring collaborations across several research groups.

The authors have also highlighted several issues with regards to study design and analyses that are common to geneassociation studies, including candidate gene analysis and GWAS, and that perhaps could pose more problems as larger samples are collected for GWAS.

First, accurately defining the phenotype is critical for any gene-association study. If infection and severe sepsis susceptibility and outcomes are due to gene-environment interaction, then determining the causal organism would be important. Limitations of current microbiologic techniques, however, allow an accurate determination of the causative 
agent in only a small number of cases. For instance, consider a case-control design of Gram-positive organisms causing community-acquired pneumonia with population-based controls used to assess pneumonia susceptibility. In such a study, we might exclude a large number of cases that are culture-negative and include only those with severe pneumonia who are culture-positive. An alternative approach would be to include cases that are identified based on clinical syndromes, such as severe sepsis, to identify genetic variation within proteins that are likely to play an important role in the broader host response to infection, regardless of the causative agent.

Second, choosing appropriate controls is important as we assess the role of genetic variants in the spectrum of infection and severe sepsis susceptibility. For example, comparing the distribution of the genetic variant in severe sepsis cases and healthy, population-based control individuals may be confounded by the effect of the genetic variant on infection susceptibility.

Third, the population admixture could often lead to false positive results due to association between subgroups of the population and the phenotype [4].

Finally, an important limitation of any association study is that it cannot establish a cause-effect relationship.

Functional studies can be conducted to understand how genetic variants alter the host response to infection and pose special challenges. These studies are usually conducted either in vitro using cell culture or in vivo using transgenic rodents, either with knockouts to assess the effect of removing the gene or with knockins to assess the affect of adding the gene. While both of these methods can provide useful information to help assess the functional effects of a polymorphism in humans, there are significant limitations to translating this work from the bench to the bedside.

In conclusion, new technology such as GWAS has the potential to improve our understanding of genetics of sepsis. Large studies will have to be conducted, however, to effectively utilize this technology. Issues that are common to gene-association studies should not be ignored, especially since larger, multicenter, and collaborative efforts will be required to design GWAS studies to understand the genetic determinants of sepsis.

\section{Competing interests}

The authors declare that they have no competing interests

\section{References}

1. Sutherland AM, Walley KR: Bench-to-bedside review: Association of genetic variation with sepsis. Crit Care 2009, 13:210.

2. Zeggini E, Scott LJ, Saxena R, Voight BF, Marchini JL, Hu T, de Bakker PI, Abecasis GR, Almgren P, Andersen G, Ardlie K, Bostrom KB, Bergman RN, Bonnycastle LL, Borch-Johnsen K,
Burtt NP, Chen H, Chines PS, Daly MJ, Deodhar P, Ding CJ, Doney ASF, Duren WL, Elliott KS, Erdos MR, Frayling TM, Freathy RM, Gianniny L, Grallert H, Grarup N, et al.: Meta-analysis of genome-wide association data and large-scale replication identifies additional susceptibility loci for type 2 diabetes. Nat Genet 2008, 40:638-645.

3. The Wellcome Trust Case Control Consortium: Genome-wide association study of 14,000 cases of seven common diseases and 3,000 shared controls. Nature 2007, 447:661-678.

4. Cardon LR, Palmer LJ: Population stratification and spurious allelic association. The Lancet 2003, 361:598-604. 